1 Universidade Federal de Mato Grosso (UFMT), Instituto de Ciências Exatas e Naturais (Icen), Curso de Graduação em Enfermagem, Campus Universitário de Rondonópolis Rondonópolis (MT), Brasil. lorenaenfermeira@hotmail. com

2 Universidade Federal de Mato Grosso (UFMT), Instituto de Saúde Coletiva (ISC), Programa de PósGraduação em Saúde Coletiva (PPGSC) - Cuiabá (MT), Brasil.

\section{Avaliação da qualidade da Estratégia de Saúde da Família em um município de porte médio}

\author{
Quality evaluation of the Family Health Strategy in a medium-sized \\ municipality
}

Lorena Araujo Ribeiro', João Henrique Gurtler Scatena²

DOI: 10.1590/0103-11042019S710

RESUMO O estudo objetivou avaliar Unidades de Saúde da Família (USF) frente aos atributos essenciais e derivados da Atenção Primária à Saúde (APS). Pesquisa avaliativa, quantitativa, com delineamento transversal, realizada em 35 USF de um município de médio porte de Mato Grosso. Os dados foram levantados mediante aplicação de questionário sociodemográfico e do Primary Care Assessment Tool (PCATool) junto a 397 usuários adultos e 59 profissionais dos referidos serviços. Os resultados revelaram que os usuários eram majoritariamente do sexo feminino e de baixa escolaridade. Quanto aos profissionais, observou-se elevada rotatividade, com tempo de lotação inferior a dois anos, e predomínio de indivíduos sem especialização em APS. Acerca do desempenho dos serviços na perspectiva dos usuários, todos os atributos foram avaliados como insatisfatórios, exceto Utilização e Sistema de Informação, que apresentaram escore acima do valor de corte $(6,6)$. Contrariamente, na opinião dos profissionais, todos os atributos foram avaliados como satisfatórios, com exceção da Acessibilidade, que obteve escore 5,28. Os baixos valores para a maioria dos atributos, obtidos sob a ótica de quem utiliza o serviço, evidenciam a dificuldade das USF na efetivação dos atributos da APS e impõem a necessidade de reflexões acerca das barreiras estruturais e organizacionais presentes nos serviços.

PALAVRAS-CHAVE Atenção Primária à Saúde. Estratégia Saúde da Família. Avaliação em saúde. Avaliação de serviços de saúde.

ABSTRACT The study aimed at evaluating Family Health Units (FHU) considering the essential attributes and derived from Primary Health Care (PHC). It's a evaluative, quantitative research with a cross-sectional design, carried out in 35 FHU of a medium-sized municipality of Mato Grosso. Data were collected using a sociodemographic questionnaire and the Primary Care Assessment Tool (PCATool) involving 397 adult users and 59 professionals from these services. The results reveal that users were mostly female and of low education level. As for the professionals, there was a high turnover rate, with a crowding time of less than two years, and a predominance of individuals with no specialization in PHC. Regarding the performance of the services from the perspective of the users, all attributes were evaluated as unsatisfactory, except Use and Information System, which presented scores above the cutoff value (6.6). In contrast, in the opinion of the professionals, all the attributes were evaluated as satisfactory, except for Accessibility, that obtained score 5.28. The low values for most of the attributes, obtained from the perspective of those who use the service, highlight the difficulty of the FHU in achieving the attributes of the PHC and impose the need for reflections on the structural and organizational barriers present in the services.

KEYWORD Primary Health Care. Family Health Strategy. Health evaluation. Health services research. 


\section{Introdução}

No Brasil, a reorientação do sistema de saúde para a Atenção Primária à Saúde (APS) ganha força a partir da década de 1990, com o fomento de ações de caráter preventivo e investimentos em propostas assistenciais básicas que visavam especialmente à promoção da saúde' $\mathbf{1}^{\mathbf{1}}$. Essas iniciativas materializaram-se com a implantação do Programa de Agentes Comunitários de Saúde (Pacs) e foram ampliadas com a criação do Programa de Saúde da Família (PSF), em 19942,3. É posteriormente, com a Estratégia Saúde da Família (ESF), que a APS brasileira assume sua versão mais abrangente, passando a ser compreendida como uma estratégia de reorientação de modelo de atenção. Operacionalizada pelo trabalho em equipe dirigido às populações de territórios delimitados, a ESF tem a responsabilidade de promover uma atenção continuada e integral, pautada nos princípios de universalidade, acessibilidade, vínculo, responsabilização, humanização, equidade e participação social ${ }^{4,1}$.

A intensificação de investimentos na APS nas últimas décadas, especialmente após a implantação da ESF, produziu impacto positivo em importantes indicadores de saúde, como a redução da taxa de mortalidade infantil, a diminuição das internações por condições sensíveis à APS, a elevação do acompanhamento pré-natal e o aumento expressivo da cobertura populacional por essa modalidade de serviço ${ }^{5}$. Especificamente sobre a elevação da cobertura, cabe ressaltar que o seu ritmo ocorreu de maneiras distintas, variando segundo a região e o porte populacional dos municípios ${ }^{6}$.

Na década de 2000, evidenciou-se que a meta proposta pelo governo federal de ampliar a provisão de equipes de saúde da família em pequenas cidades, principalmente naquelas com baixo Índice de Desenvolvimento Humano (IDH), havia sido alcançada. Contudo, em municípios de médio porte (aqueles com mais de 100 mil habitantes), observava-se ainda baixa aderência à ESF, relacionada, entre outros aspectos, à maior concentração demográfica, a especificidades de agravos de saúde de grandes centros populacionais, ao elevado grau de exclusão de acesso aos serviços de saúde e, também, a certa desarticulação e má distribuição da rede de atenção à saúde ${ }^{7,8}$. Diante desse contexto, em 2003, foi celebrado, entre o Ministério da Saúde e o Banco Interamericano de Reconstrução e Desenvolvimento (Bird), o Programa de Expansão e Consolidação da Saúde da Família (Proesf). Renovado em 2009, esse projeto teve por finalidade fomentar a expansão da cobertura, qualificação e consolidação da ESF nos municípios com população superior a 100 mil habitantes?.

O Proesf e outras iniciativas governamentais de fomento à expansão e sustentabilidade da ESF compõem um processo político que vem se fortalecendo gradativamente nos últimos anos, repercutindo, principalmente, no aumento da cobertura populacional por essa modalidade ${ }^{10,11}$. Todavia, o crescimento quantitativo da ESF parece não ser garantia de incremento na qualidade de ações e serviços nelas desenvolvidos, uma vez que estudos apontam que ainda é possível identificar nesses espaços certo privilégio do manejo clínico de condições agudas, em detrimento das crônicas e do desenvolvimento de ações de promoção e acompanhamento dos usuários 2,12.

Diante da complexidade e da relevância das ESF e dos desafios que se impõem na implementação dessa proposta, é imprescindível a adoção de estratégias para monitoramento e avaliação da ESF com a utilização de ferramentas que permitam perceber os alcances e limites impressos nesse processo ${ }^{2}$.

A literatura descreve uma variedade de ferramentas dedicadas à avaliação da qualidade de serviços, contudo, poucas possibilitam a avaliação da APS a partir dos atributos que a definem e qualificam, como o faz o Primary Care Assessment Tool - PCATool (Instrumento de Avaliação da Atenção Primária) ${ }^{\mathbf{1 3}}$.

O PCATool foi projetado para mensurar a presença e a extensão dos atributos essenciais (acesso de primeiro contato, longitudinalidade, integralidade e coordenação da atenção) e 
derivados (centralidade na família, orientação comunitária e competência cultural) da APS e tem sido reconhecido por conferir rigor e qualidade ao processo de identificação e distinção de modelos de atenção ambulatorial ${ }^{13-17}$. Por se tratar de um instrumento de avaliação validado internacionalmente e apresentar versões aplicáveis aos diferentes atores envolvidos na APS (usuários adultos, responsáveis por crianças e profissionais), favorece a produção de evidências acerca da efetividade da APS sob diferentes perspectivas, oferecendo subsídios consistentes para o planejamento e a execução de políticas públicas, além de oportunizar comparabilidade entre diferentes realidades nacionais e internacionais ${ }^{\mathbf{1 8}}$.

Diante do exposto, este estudo teve como objetivo avaliar Unidades de Saúde da Família (USF) de um município de médio porte, na perspectiva de usuários adultos e profissionais, buscando analisar a qualidade da APS, frente a seus atributos essenciais e derivados.

\section{Material e métodos}

Estudo avaliativo, de abordagem quantitativa e delineamento transversal, realizado em USF do município de Rondonópolis, o terceiro mais populoso do estado de Mato Grosso, com importante papel no processo de regionalização nessa Unidade da Federação (UF). É o município sede da região Sul Mato-grossense de Saúde - a maior do estado em número de municípios $^{19}$, destacando-se, ainda, por concentrar serviços de grande aporte tecnológico e por sediar o Consórcio Intermunicipal Sul Mato-Grossense, constituindo-se, assim, referência para diversas especialidades médicas aos dezenove municípios da região.

A rede de APS de Rondonópolis organiza-se em cinco distritos sanitários (Norte, Sul, Leste, Oeste e Centro Oeste) e dispõe, além de USF, de unidades básicas tradicionais (UBS), com e sem estratégia de Agentes Comunitários de Saúde (ACS). A pesquisa ocorreu em 35 USF da zona urbana do município, todas com mais de dois anos ininterruptos de funcionamento.

Participaram desta investigação usuários e profissionais dos referidos serviços. Os critérios para inclusão dos profissionais foram: ter formação em nível superior em enfermagem ou medicina; estar alocado nas USF selecionadas por pelo menos seis meses; não estar de férias ou afastado de suas atividades durante o período de coleta; e aceitar participar da pesquisa.

Para o grupo de respondentes da categoria usuários, utilizou-se o procedimento amostral probabilístico estratificado segundo distrito sanitário, cujo cálculo adotou como pressupostos: nível de confiança de $95 \%$, erro amostral de $5 \%$ e proporção esperada de 0,50 . Respeitandose o planejamento amostral, foram selecionados indivíduos adultos de ambos os sexos que atendiam aos seguintes requisitos: idade igual ou superior a 18 anos; ter tido atendimento nos últimos seis meses, realizado por médico ou enfermeiro; possuir cadastro familiar na USF; e aceitar participar da pesquisa. Foram excluídos os usuários submetidos exclusivamente a atendimento odontológico, sem condições cognitivas e de comunicação para responder aos questionamentos da entrevista, ou que relatassem ser aquele o primeiro atendimento na unidade estudada.

A coleta de dados ocorreu de julho a agosto de 2017, sendo utilizados um questionário sociodemográfico, específico para cada uma das duas categorias de entrevistados, e o PCAtoolBrasil, nas versões adulto e profissional.

O PCATool tem sido descrito na literatura como um instrumento válido e fidedigno para avaliar, sob perspectivas distintas, a qualidade da Atenção Primária à Saúde em diferentes serviços, sendo sugerido como ferramenta preferencial para verificação do grau de orientação destes aos atributos da Atenção Primária ${ }^{13,16,17}$.

A versão PCAtool-Brasil adulto é constituída de 87 itens, e a profissional de 77 , ambas divididas em componentes relacionados aos atributos essenciais (Acesso de Primeiro Contato, Longitudinalidade, 
Coordenação e Integralidade) e aos atributos derivados (Orientação Familiar e Orientação Comunitária). As respostas são estruturadas numa escala do tipo Likert, cujos valores possíveis para cada item do questionário são: 'com certeza sim' (valor $=4$ ), 'provavelmente sim' (valor=3), 'provavelmente não' (valor=2), 'com certeza não' (valor=1) e 'não sei/ não lembro' (valor=9, não considerado no cálculo de média do item). O escore para cada um dos atributos ou seus componentes é calculado através da média aritmética simples dos valores das respostas dos itens que os compõem ${ }^{15}$.

Além dos escores de cada atributo e seus componentes, calculou-se o escore essencial (resultante da soma dos escores médios dos atributos essenciais, dividida pelo número total de componentes) e o escore geral (medido pela soma dos escores médios dos atributos essenciais e derivados dividido pelo número total de componentes) ${ }^{15}$. Os valores alcançados foram ainda transformados para uma escala de 0 a 10, aplicando-se a fórmula: $\mathrm{E}=$ [(escore $-1) \times 10] / 3$, onde o escore com valor médio $\geq$ 6,6 indica qualidade satisfatória e $<6,6$ sugere qualidade insatisfatória ${ }^{20}$.

Os dados foram registrados (em dupla digitação) e analisados utilizando-se o pacote estatístico Epi Info 2000 versão 3.3.2. Para elaboração e organização do banco, empregou-se o software Microsoft Excel.

Os dados relativos à caracterização dos usuários e profissionais foram expressos em frequência absoluta e percentual; aqueles originários da aplicação do PCATool foram apresentados no formato de escore médio, calculado para cada atributo e seus componentes, além dos escores essencial e geral. Os atributos essenciais com avaliação desfavorável foram detalhados segundo seus itens, apresentando-se, ainda, a frequência relativa das respostas para alguns deles.

$\mathrm{O}$ estudo obedeceu aos preceitos da Resolução nº 466/2012 do Conselho Nacional de Saúde, sendo aprovado pelo Comitê de Ética em Pesquisa por meio da Plataforma Brasil, sob o registro CAAE de n ${ }^{\circ}$ 68354617.3.0000.5541.

\section{Resultados}

Foram entrevistados 397 usuários adultos e 59 profissionais, de 35 USF. Entre os usuários, observou-se o predomínio de pessoas do sexo feminino $(75,57 \%)$, casadas $(45,59 \%)$, autorreferidas de cor parda $(58,19 \%)$ e com idade média de 48,46 anos. A média de idade das mulheres foi de 47,09 anos, e dos homens 52,70 anos. Quanto à escolaridade, a maior parte dos usuários $(42,42 \%)$ afirmou ter apenas o ensino fundamental incompleto. Evidenciou-se que $68,01 \%$ dos entrevistados possuíam famílias compostas por até três pessoas, e a maioria dos indivíduos $(57,83 \%)$ referiu renda familiar de até dois salários mínimos.

No que se refere aos profissionais, destacaram-se as seguintes caraterísticas: 30 enfermeiros e 29 médicos, predomínio do sexo feminino (74,58\%), idade entre 20 e 39 anos (74,57\%), casados (61,02\%) e brancos (54,24\%). Mais da metade dos profissionais concluiu o curso de graduação na região Centro-Oeste $(61,02 \%)$, e quase metade $(52,54 \%)$ tinha se graduado há menos de cinco anos.

Quanto à atuação profissional, 61,02\% dos participantes possuíam, no máximo, 2 anos de experiência prática na atenção primária, e $62,71 \%$ relataram exercer suas atividades na USF de lotação por um período inferior a 1 ano. Com relação à formação complementar, $66,67 \%$ dos enfermeiros referiram algum tipo de pós-graduação, sendo a maioria em áreas não relacionadas à APS. Quanto aos médicos, observou-se equilíbrio entre o percentual de profissionais sem (51,72\%) e com pós-graduação (48,28\%), neste caso, com predomínio de especialização em outras áreas que não a atenção primária.

Sobre a avaliação das USF frente aos atributos essenciais e derivados da APS, destaca-se a diferença entre a percepção dos usuários e dos profissionais com relação a praticamente todos os escores, o que se reflete, também, nos escores essencial e geral (gráfico 1). 
Gráfico 1. Escores médios de cada atributo da Atenção Primária à Saúde e escores essencial e geral, segundo avaliação de usuários e profissionais de Unidades de Saúde da Família, Rondonópolis-MT, 2018

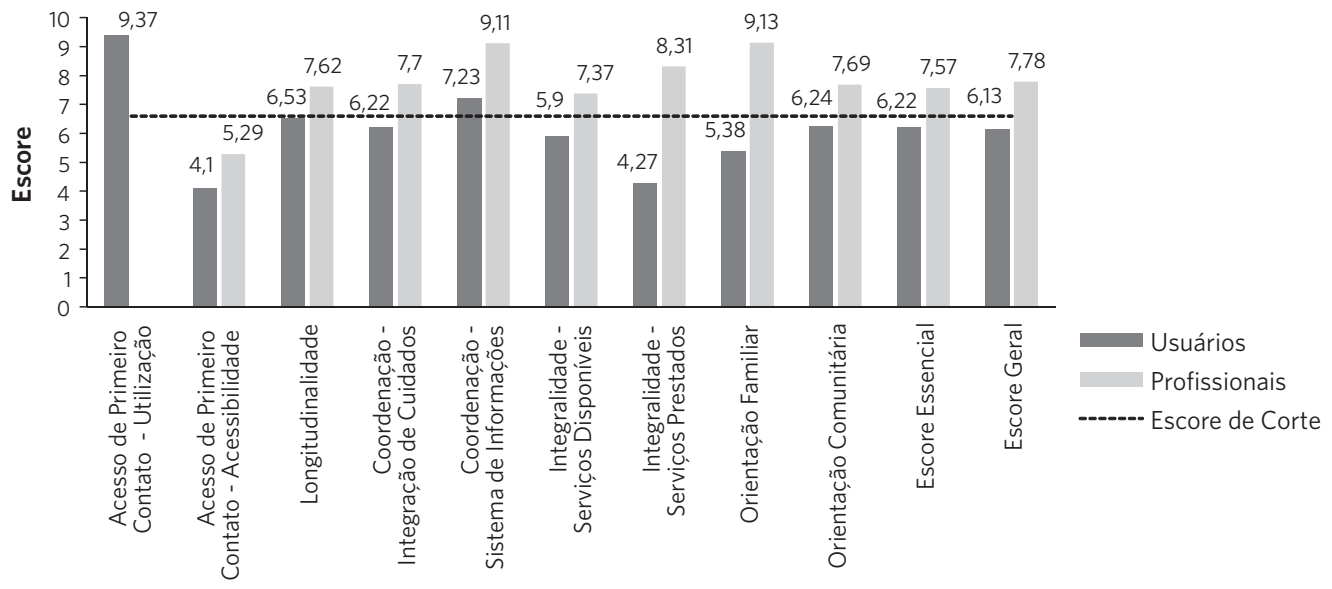

Fonte: Elaborado própria a partir das respostas ao questionário PCATool - versão adulto e profissionais.

Segundo os usuários, apenas dois componentes dos atributos essenciais foram considerados satisfatórios: Acesso de Primeiro Contato - Utilização, que apresentou uma pontuação considerada elevada (9,37), e Coordenação Sistema de Informação, que alcançou o escore de 7,23. Todos os demais atributos essenciais e seus componentes apresentaram valores abaixo do aceitável. Ambos os atributos derivados - Orientação Familiar e Orientação Comunitária - também tiveram avaliação desfavorável, e, como consequência, os escores essencial e geral redundaram em valores insatisfatórios, 6,22 e 6,13, respectivamente.

Na perspectiva dos profissionais, a maioria dos atributos essenciais e derivados da APS foi avaliada como satisfatória, divergindo dos usuários. Apenas os componentes Acesso de Primeiro Contato - Acessibilidade $(5,29)$ e Coordenação - Sistema de Informação $(9,11)$ ficaram na mesma qualificação aferida pelos usuários, ainda que com valores maiores.

Para os profissionais, excetuando o Primeiro Contato - Acessibilidade, todos os demais atributos foram avaliados positivamente, o que se refletiu nos escores essencial $(7,57)$ e geral $(7,78)$.

Para melhor destacar algumas das potencialidades e dificuldades na operacionalização dos serviços de APS com qualidade, relata-se o detalhamento das respostas dos entrevistados a cada item dos atributos essenciais avaliados como insatisfatórios.

Para as duas categorias de respondentes (profissionais e usuários), as principais limitações relacionadas ao atributo Acesso de Primeiro Contato - Acessibilidade foram: funcionamento dos serviços limitados aos períodos matutino e vespertino, de segunda a sexta-feira, sem a oferta de expedientes alternativos; ausência de suporte assistencial presencial ou remoto (via telefone) disponibilizado pela USF, quando esta se encontra fechada. A maioria dos itens que abordavam acesso ao serviço durante o horário regular de funcionamento foi bem avaliada pelos usuários. Foram eles: organização para a marcação de consultas; atendimento no mesmo dia; atendimento médico nas ocasiões necessárias; aconselhamento rápido via telefone. Apenas o item que faz referência ao tempo dedicado a aguardar atendimento médico/de enfermagem foi avaliado de maneira negativa, expressando que o usuário precisa aguardar mais de trinta minutos para ser atendido.

A avaliação do atributo Longitudinalidade 
(tabela 1) denotou que os profissionais têm conhecimento da dimensão clínica das pessoas atendidas, mas ele é limitado quanto às suas especificidades sociais, financeiras e de trabalho. Já o domínio de informações sobre os problemas mais relevantes para o usuário apresentou avaliação um pouco melhor. Os itens relacionados à comunicação entre usuário e profissional foram avaliados positivamente, exceto quando esta não é presencial (D4).

Tabela 1. Distribuição proporcional das respostas dos usuários adultos aos itens do atributo Longitudinalidade, Rondonópolis-MT, 2018. $(n=397)$

\begin{tabular}{|c|c|c|c|c|c|}
\hline \multirow[t]{2}{*}{ Item } & \multirow[t]{2}{*}{ Aspectos avaliados } & 1 & 2 & 3 & 4 \\
\hline & & $\%$ & $\%$ & $\%$ & $\%$ \\
\hline D1 & É o mesmo profissional que atende o usuário rotineiramente & 8,56 & 6,80 & 12,85 & 71,79 \\
\hline D2 & O profissional compreende as perguntas realizadas & 1,26 & 1,76 & 16,12 & 80,86 \\
\hline D3 & O profissional responde às perguntas de maneira acessível & 1,76 & 1,76 & 10,33 & 86,15 \\
\hline D4 & $\begin{array}{l}\text { O usuário pode telefonar e falar com o profissional para escla- } \\
\text { recer dúvidas }\end{array}$ & 45,84 & 22,42 & 13,60 & 18,14 \\
\hline D5 & $\begin{array}{l}\text { O profissional disponibiliza tempo suficiente para o usuário } \\
\text { expor suas preocupações ou problemas }\end{array}$ & 7,56 & 4,28 & 9,57 & 78,59 \\
\hline D6 & $\begin{array}{l}\text { O usuário se sente à vontade para falar de suas preocupações } \\
\text { ou problemas com o profissional }\end{array}$ & 6,55 & 3,02 & 8,82 & 81,61 \\
\hline D7 & $\begin{array}{l}\text { O profissional conhece o usuário mais como pessoa do que } \\
\text { somente como alguém com um problema de saúde }\end{array}$ & 44,08 & 17,63 & 12,09 & 26,20 \\
\hline D8 & O profissional sabe das pessoas que residem com usuário & 41,31 & 12,85 & 6,80 & 39,04 \\
\hline D9 & $\begin{array}{l}\text { O profissional sabe quais problemas são mais importantes } \\
\text { para o usuário }\end{array}$ & 28,46 & 15,62 & 16,88 & 39,04 \\
\hline D10 & O profissional conhece a história clínica do usuário & 22,42 & 11,84 & 16,37 & 49,37 \\
\hline D11 & $\begin{array}{l}\text { O profissional sabe a respeito do seu trabalho ou emprego do } \\
\text { usuário }\end{array}$ & 50,12 & 13,10 & 5,29 & 31,49 \\
\hline D12 & $\begin{array}{l}\text { O profissional tem conhecimento acerca de dificuldades do } \\
\text { usuário para obter ou pagar por medicamentos }\end{array}$ & 42,31 & 18,39 & 10,08 & 29,22 \\
\hline D13 & $\begin{array}{l}\text { O profissional tem conhecimento a respeito das medicações } \\
\text { utilizadas pelo usuário }\end{array}$ & 16,88 & 8,31 & 8,82 & 65,99 \\
\hline D14 & $\begin{array}{l}\text { O usuário deseja ser atendimento em outro serviço de saúde } \\
\text { caso isso lhe represente mais facilidade }\end{array}$ & 61,20 & 12,85 & 6,05 & 19,90 \\
\hline
\end{tabular}

Fonte: Elaboração própria a partir das respostas ao questionário PCATool - versão adulto.

1- Com certeza não; 2- Provavelmente não; 3- Provavelmente sim; 4- Com certeza sim.

$\mathrm{O}$ atributo Coordenação apresentou escore médio insatisfatório apenas para o componente Integração de Cuidados, destacando-se que tal avaliação se deu entre os 324 usuários $(81,61 \%)$ que, durante o período de acompanhamento no serviço, necessitaram e foram submetidos a consulta com especialista.

O detalhamento desse atributo mostrou que as equipes das USF eram responsáveis pela maioria dos encaminhamentos a atendimentos especializados, auxiliavam no agendamento das consultas e ofertavam informação escrita ao especialista e/ou serviço especializado (tabela 2). Todavia, no tocante a este último aspecto, chama a atenção o elevado percentual de respostas desfavoráveis (46,29\%). A possibilidade de o usuário opinar na seleção do serviço especializado disponível para seu problema de saúde e a investigação/valorização das suas experiências com os atendimentos nesses espaços receberam avaliação desfavorável. 
Tabela 2. Distribuição proporcional das respostas dos usuários adultos aos itens do atributo Coordenação - Integração de Cuidados, Rondonópolis-MT, 2018. $\left(n=324^{\star}\right)$

\begin{tabular}{|c|c|c|c|c|c|}
\hline \multirow[t]{2}{*}{ Item } & \multirow[t]{2}{*}{ Aspectos avaliados } & 1 & 2 & 3 & 4 \\
\hline & & $\%$ & $\%$ & $\%$ & $\%$ \\
\hline E2 & $\begin{array}{l}\text { O serviço sugeriu/ encaminhou o usuário à consulta com } \\
\text { especialista }\end{array}$ & 14,52 & 1,23 & 1,23 & 83,02 \\
\hline E3 & $\begin{array}{l}\text { O serviço tem conhecimento das consultas realizadas com } \\
\text { especialistas }\end{array}$ & 6,17 & 2,48 & 5,86 & 85,49 \\
\hline E4 & $\begin{array}{l}\text { O profissional discute com o usuário os diferentes serviços } \\
\text { onde poderia obter atendimento para o problema de saúde }\end{array}$ & 59,25 & 9,26 & 3,40 & 28,09 \\
\hline E5 & $\begin{array}{l}\text { Alguém do serviço auxilia o usuário com a marcação da con- } \\
\text { sulta especializada }\end{array}$ & 14,20 & 1,54 & 2,16 & 82,10 \\
\hline E6 & $\begin{array}{l}\text { O profissional oferta informação escrita para o especialista, a } \\
\text { respeito do motivo da consulta }\end{array}$ & 37,96 & 8,33 & 4,94 & 48,77 \\
\hline E7 & $\begin{array}{l}\text { O serviço tem conhecimento dos resultados da consulta com } \\
\text { o especialista }\end{array}$ & 23,15 & 10,19 & 8,33 & 58,33 \\
\hline E8 & $\begin{array}{l}\text { Depois da consulta com especialista, o profissional conversa } \\
\text { com o usuário sobre o que aconteceu durante o atendimento }\end{array}$ & 50,00 & 7,41 & 4,01 & 38,58 \\
\hline E9 & $\begin{array}{l}\text { O profissional se interessa pela qualidade do cuidado ofertado } \\
\text { ao usuário durante o atendimento especializado }\end{array}$ & 54,01 & 6,79 & 4,01 & 35,19 \\
\hline
\end{tabular}

Fonte: Elaboração própria a partir das respostas ao questionário PCATool - versão adulto.

1- Com certeza não; 2- Provavelmente não; 3- Provavelmente sim; 4- Com certeza sim.

*Total de pessoas que afirmaram ter sido consultadas em serviço especializado no período em que estavam em acompanhamento na equipe de saúde da família pesquisada.

A Integralidade - Serviços Disponíveis, que apresentou baixo escore $(5,90)$, alude à experiência do usuário com as intervenções disponibilizadas pelo serviço, mesmo que ainda não as tenham utilizado. A avaliação desse atributo considera as ações, as orientações e os procedimentos disponíveis (tabela 3).

Tabela 3. Distribuição proporcional das respostas dos usuários adultos ao atributo Integralidade - Serviços Disponíveis, Rondonópolis-MT, 2018. ( $n=397)$

\begin{tabular}{|c|c|c|c|c|c|c|}
\hline \multirow[t]{2}{*}{ Item } & \multirow[t]{2}{*}{ Aspectos avaliados } & 1 & 2 & 3 & 4 & 9 \\
\hline & & $\%$ & $\%$ & $\%$ & $\%$ & $\%$ \\
\hline & \multicolumn{6}{|l|}{ Rol de Ações } \\
\hline G2 & $\begin{array}{l}\text { Inclusão de famílias em programa de assistência social/benefí- } \\
\text { cios sociais }\end{array}$ & 31,99 & 18,89 & 14,36 & 34,26 & 0,50 \\
\hline G3 & Programa de suplementação nutricional & 42,07 & 38,29 & 8,06 & 10,58 & 1,01 \\
\hline G4 & Imunizações & 1,01 & 0,50 & 2,52 & 95,97 & - \\
\hline G5 & Avaliação de saúde bucal & 21,91 & 4,53 & 2,78 & 70,78 & - \\
\hline G7 & Planejamento familiar ou métodos anticoncepcionais & 4,53 & 3,53 & 9,57 & 82,12 & 0,25 \\
\hline G8 & Aconselhamento/tratamento para o uso prejudicial de drogas & 13,60 & 38,54 & 17,63 & 29,22 & 1,01 \\
\hline G11 & Aconselhamento/solicitação de teste anti-HIV & 6,30 & 21,66 & 16,88 & 54,41 & 0,75 \\
\hline G12 & Identificação/avaliação de problemas auditivos & 26,45 & 43,07 & 17,38 & 12,09 & 1,01 \\
\hline G13 & Identificação/avaliação de problemas visuais & 33,75 & 38,29 & 14,36 & 12,59 & 1,01 \\
\hline \multirow[t]{2}{*}{ G18 } & Cuidados pré-natais. & 1,26 & 1,76 & 7,81 & 89,17 & - \\
\hline & Rol de Orientações & & & & & \\
\hline G1 & Nutrição ou dieta & 24,94 & 22,92 & 12,85 & 39,04 & 0,25 \\
\hline G9 & Aconselhamento para problemas de saúde mental & 13,85 & 26,20 & 19,14 & 40,05 & 0,76 \\
\hline G17 & Aconselhamento sobre como parar de fumar & 16,12 & 30,98 & 19,90 & 32,49 & 0,51 \\
\hline
\end{tabular}




\begin{tabular}{|c|c|c|c|c|c|c|}
\hline \multicolumn{7}{|c|}{ Tabela 3. (cont.) } \\
\hline G20 & Aconselhamento sobre alterações próprias do envelhecimento & 22,17 & 21,41 & 19,90 & 36,02 & 0,50 \\
\hline G21 & Cuidados domiciliares & 10,08 & 12,59 & 17,38 & 59,19 & 0,76 \\
\hline \multirow[t]{2}{*}{$\mathrm{G} 22$} & Situações críticas de saúde & 17,13 & 25,19 & 24,69 & 32,49 & 0,50 \\
\hline & Rol de Procedimentos & & & & & \\
\hline G6 & Tratamento dentário & 22,42 & 4,53 & 4,53 & 68,52 & - \\
\hline G10 & Realização de sutura & 24,18 & 32,49 & 15,87 & 26,70 & 0,76 \\
\hline G14 & Colocação de tala & 43,83 & 37,28 & 10,08 & 8,31 & 0,50 \\
\hline G15 & Remoção de verrugas & 50,88 & 37,78 & 5,29 & 5,29 & 0,76 \\
\hline G16 & Exame preventivo para câncer de colo de útero & 0,76 & 3,27 & 4,28 & 91,69 & - \\
\hline G19 & Remoção de unha encravada & 36,52 & 43,07 & 13,85 & 6,05 & 0,51 \\
\hline
\end{tabular}

Fonte: Elaboração própria a partir das respostas ao questionário PCATool - versão adulto.

1- Com certeza não; 2- Provavelmente não; 3- Provavelmente sim; 4- Com certeza sim; 9- Não sabe/não lembra.

No elenco de ações, compreendido como tudo aquilo que o serviço implementa em função de algo, destacaram-se negativamente: a disponibilização/inclusão em programas de assistência social e programas de suplementação nutricional; o aconselhamento/ tratamento para o uso prejudicial de drogas; a identificação/avaliação de problemas visuais e auditivos. As ações diretamente relacionadas a programas ministeriais, no âmbito da ESF, apresentaram avaliações positivas: imunização; avaliação de saúde bucal; planejamento familiar e cuidados pré-natais; e aconselhamento/solicitação de teste anti-HIV.

Todos os itens alusivos às orientações foram avaliados favoravelmente por mais de metade dos usuários. Todavia, com exceção das orientações relacionadas aos cuidados domiciliares, um elevado percentual de usuários (entre 40\% e 47\%) as avaliou desfavoravelmente. Quanto ao rol de procedimentos, quatro dos seis itens que o compõem foram considerados indisponíveis pelos entrevistados. Apenas tratamento dentário e exame preventivo de colo de útero foram reconhecidos como disponíveis.

O atributo Integralidade - Serviços Prestados apresentou escore médio insatisfatório $(4,27)$. Essa dimensão relaciona-se aos assuntos abordados durante atendimento médico ou realizado pelo enfermeiro. A sumarização dos itens que a compõem evidenciou que apenas quatro dos treze constantes foram avaliados de maneira positiva: solicitação de exames; orientações relativas à medicação utilizada; aconselhamento sobre alimentação saudável e padrões de repouso; aconselhamento sobre prática adequada de atividade física (tabela 4). Todos os demais foram avaliados desfavoravelmente.

Tabela 4. Distribuição percentual das respostas dos usuários adultos ao atributo Integralidade - Serviços Prestados, RondonópolisMT, 2018. $(n=397)$

\begin{tabular}{llrrrr}
\hline Item & Aspectos avaliados & $\mathbf{1}$ & $\mathbf{2}$ & $\mathbf{3}$ & $\mathbf{4}$ \\
\hline H1 & Aconselhamento sobre alimentação saudável e repouso & \% & \% & \% & \% \\
\hline H2 & Aconselhamento sobre medidas de segurança no domicílio & 29,72 & 2,27 & 4,28 & 63,73 \\
H3 & Aconselhamento sobre medidas de segurança no trânsito & 47,10 & 6,55 & 5,54 & 40,81 \\
H4 & Orientações sobre gerenciamento de conflitos familiares & 71,54 & 7,81 & 3,02 & 17,63 \\
H5 & Aconselhamento sobre a prática de atividade física & 72,04 & 11,34 & 3,02 & 13,60 \\
H6 & Solicitação de exames laboratoriais básicos & 25,69 & 4,03 & 5,54 & 64,74 \\
H7 & Avaliação e orientação acerca da medicação utilizada & 12,85 & 1,76 & 3,78 & 81,61 \\
\hline
\end{tabular}




\begin{tabular}{lllllr}
\hline Tabela 3. (cont.) & & & & \\
\hline H8 & Investigação quanto à exposição a substâncias tóxicas/inflamáveis & 64,99 & 8,82 & 4,28 & 21,91 \\
H9 & Orientação sobre acondicionamento seguro de arma de fogo no domicílio & 93,95 & 4,03 & - & 2,02 \\
H10 & Medidas de prevenção de queimaduras & 68,77 & 7,30 & 4,03 & 19,90 \\
H11 & Medidas para prevenção de quedas. & 66,75 & 6,80 & 3,53 & 22,92 \\
H12 & Medidas de prevenção de osteoporose ou desgaste ósseo* & 59,67 & 6,00 & 5,00 & 29,33 \\
H13 & Orientações relacionadas à menstruação ou menopausa* & 48,00 & 3,67 & 6,67 & 41,66 \\
\hline
\end{tabular}

Fonte: Elaboração própria a partir das respostas ao questionário PCATool - versão adulto.

1- Com certeza não; 2- Provavelmente não; 3- Provavelmente sim; 4- Com certeza sim.

*Pergunta realizada somente às mulheres $(n=300)$.

\section{Discussão}

Na caracterização sociodemográfica dos usuários, destacou-se a maior proporção de pessoas do sexo feminino e de baixa escolaridade (ensino fundamental incompleto), corroborando resultados de estudos semelhantes desenvolvidos no Brasil21,22. Com relação aos profissionais, observou-se elevada rotatividade profissional nas unidades e predomínio de indivíduos com menos de cinco anos de conclusão de curso de graduação, sem especialização na área da APS, com pouco tempo de atuação em serviços dessa natureza (máximo de dois anos).

A baixa formação profissional em APS foi igualmente encontrada em pesquisas análogass $^{23,24}$. Cabe ressaltar que o incremento da qualidade dos serviços de APS perpassa obrigatoriamente investimentos na qualificação profissional, que pode ser enfrentada com estímulo aos programas de residência ou especializações com foco em saúde da família e comunidades 25 . Quanto à alta rotatividade profissional, o resultado pode estar relacionado ao tipo de vínculo referido pelos entrevistados, que, em sua totalidade, eram comissionados ou contratados. Pesquisas realizadas em diferentes regiões, e também em Mato Grosso, denotaram vínculos empregatícios precários entre trabalhadores de serviços de APS. Essa condição é apontada na literatura como um dos meios de controle político sobre as equipes e como uma das principais causas da insatisfação profissional, aspectos que podem constituir obstáculos na implementação de uma atenção de qualidade pautada no comprometimento e na responsabilização dos múltiplos atores 26-28.

A avaliação dos atributos, por parte dos profissionais, foi mais favorável à APS que a dos usuários, redundando em escores satisfatórios a todas as dimensões, exceto a Acessibilidade. A literatura tem verificado diferenças nas percepções desses dois grupos, geralmente com melhor avaliação por parte dos profissionais ${ }^{29-31}$. Acredita-se que essa divergência possa estar relacionada ao fato desta categoria conhecer melhor os serviços e seus dispositivos, ou, ainda, que algumas ações sejam desenvolvidas de forma inapropriada ou insuficiente à apreensão do usuário, sugerindo a necessidade de fortalecimento dos espaços de participação social nas ESF, além da adoção de estratégias que promovam maior aproximação entre os agentes nelas envolvidos ${ }^{30,31}$.

A opinião de profissionais e usuários coincidiu em todos os aspectos do componente Acesso de Primeiro Contato - Acessibilidade, e evidenciou que há obstáculos para a assistência aos indivíduos fora do horário habitual de funcionamento e quanto a alguns aspectos da organização da rotina de atendimento, já que o tempo de espera na unidade era superior a 30 minutos. Tais resultados têm sido frequentemente observados em estudos brasileiros que utilizaram o PCATool como instrumento de avaliação da APS, e, assim como na presente investigação, a Acessibilidade tem apresentado os menores escores médios, influenciando negativamente o escore essencial e o geral do município ${ }^{31-33}$. Há de se ponderar que o PCATool consiste em uma ferramenta de uso internacional que pode não atender a todas as especificidades organizacionais da Atenção 
Primária brasileira. Desse modo, para alcançar melhores desfechos nesse quesito, sugere-se a readaptação do instrumento para a realidade brasileira ou a reorganização do modelo de APS no contexto nacional, ampliando a disponibilidade dos serviços dessa natureza aos indivíduos, fortalecendo o vínculo e reduzindo a busca de serviços de pronto atendimento ${ }^{\mathbf{3 4}}$.

A avaliação da Longitudinalidade foi considerada insatisfatória apenas entre os usuários. O detalhamento desse atributo mostrou que os serviços dispõem de informação acerca da condição clínica dos usuários, que os identificam como um canal possível e favorável para abordar suas preocupações ou problemas. Entretanto, observaram-se debilidades na efetivação de vínculo pessoal com a clientela assistida, manifestas em relações pautadas prioritariamente no atendimento de necessidades de âmbito físico/ clínico e certa invisibilidade de aspectos de ordem social dos usuários como condição familiar, de trabalho, econômica e logística. Sobre as avaliações positivas acerca do domínio de informações dos problemas mais importantes para o usuário, ressalta-se que o tópico pode ter sido interpretado pelo entrevistado tanto como problemas de saúde quanto de outras ordens, haja vista que não possui especificação sobre a natureza destes no instrumento e nem no manual de aplicação.

Pesquisas anteriores dedicadas a comparar a presença e extensão dos atributos da APS entre unidades tradicionais e unidades com ESF relevou baixos escores para Longitudinalidade nas tradicionais e elevado naquelas com $\mathrm{ESF}^{35,34}$. Também em investigações que avaliaram apenas unidades com saúde da família, a Longitudinalidade foi bem avaliada, tanto por profissionais quanto por usuários ${ }^{\mathbf{2 4}, \mathbf{3 2}, 36}$. Ou seja, o fato desse atributo não ter tido uma avaliação satisfatória pelos usuários, no município em estudo, suscita uma preocupação e deve fomentar um esforço de reversão daqueles itens que mais o influenciaram, negativamente.

A Longitudinalidade presume a existência de uma fonte regular e duradoura de cuidados, independentemente da natureza do problema de saúde ou até mesmo da sua presença ${ }^{14}$. $\mathrm{O}$ vínculo estabelecido entre usuários e profissionais/ serviço é condição essencial para a implementação desse atributo. Seu alcance se dá através da confiança dos usuários nos profissionais, na responsabilização da equipe pelo cuidado e do amplo conhecimento acerca dos usuários, suas famílias e do território no qual estão inseridos ${ }^{\mathbf{1}}$. Ressalta-se que o atendimento a esse atributo não depende apenas da prática profissional. É necessário também que a gestão priorize condutas que confluam nesse propósito, como, por exemplo, garantir a oferta adequada de serviços e estratégias de fixação dos profissionais nas unidades ${ }^{37}$. Essas duas prerrogativas não se explicitaram neste estudo, haja vista que se evidenciou tanto a elevada rotatividade profissional nas unidades, provavelmente decorrente de fragilidades de vínculo trabalhista, como a acessibilidade aos serviços, dificultada pelo arranjo organizacional.

No que tange ao atributo Coordenação, a avaliação do componente Sistema de Informação foi satisfatória para as duas categorias de respondentes. Já o componente Integração de Cuidados obteve baixo escore entre os usuários e relevou que as unidades auxiliavam no agendamento de consultas em outros serviços, quando se fazia necessário; no entanto, a continuidade do cuidado era prejudicada ao não se conceder ao usuário a possibilidade de optar por uma fonte habitual de cuidado especializado entre as existentes. Outro aspecto mal avaliado referiu-se à valorização da experiência do usuário com a assistência ofertada em outros locais da rede, haja vista que as vivências e a qualidade desses atendimentos não eram abordadas após o seu retorno à unidade.

A coordenação efetiva do cuidado configura-se como uma provocação e um desafio a todos os envolvidos na operacionalização da APS. Na esfera gestora, requer a priorização de investimentos na implantação e manutenção de ferramentas que facilitem a transmissão de informações importantes dos indivíduos assistidos (como histórico de saúde, atendimentos prévios, resultados de exames etc.), além do desenvolvimento de mecanismos organizacionais que possibilitem aos usuários transitar pelos serviços e profissionais disponíveis, sem 
que haja prejuízo na continuidade de sua assistência ${ }^{1,14,34}$. No âmbito profissional local, é imperativa a garantia de espaços mais democráticos que permitam aos usuários manifestar seus desejos e julgamentos. Ademais, é essencial que se desenvolva na equipe de ESF o senso de responsabilização pelo cuidado da população assistida, reconhecendo que os eventuais encaminhamentos não se configuram como uma transferência permanente de responsabilidades, tendo em vista que a continuidade da assistência é atribuição essencial dos serviços de APS 21,38.

Outro importante atributo avaliado pelos usuários como insatisfatório foi a Integralidade, apresentando valores aquém do satisfatório tanto para o componente Serviços Prestados quanto para Serviços Disponíveis. A análise desse atributo revelou que as ESF implementavam ações (de prevenção, promoção e manutenção da saúde) ligadas, essencialmente, aos principais programas ministeriais no âmbito da APS. Entre as fragilidades identificadas, evidenciou-se que as ações executadas eram preferencialmente orientadas às demandas biológicas, contudo, com dificuldade em disponibilizar procedimentos médico-cirúrgicos ambulatoriais. No que se refere ao elenco de serviços disponíveis, observaram-se limitações relacionadas à oferta de serviços/ações que exigiam certa interdisciplinaridade ou que buscavam responder a necessidades que extrapolavam demandas de ordens físicas, como, por exemplo, as que eram motivadas pelas dimensões psíquica e/ou social dos sujeitos.

Resultados negativos para a Integralidade foram igualmente obtidos por estudos semelhantes ${ }^{20,22,39}$. Tais achados denotam que a qualificação da APS ainda corresponde a um grande desafio, apesar dos avanços na reorganização da APS no contexto brasileiro, com a implantação das ESF e da ampliação da cobertura populacional por esses novos modelos ${ }^{39,11}$. As falhas identificadas na operacionalização desse atributo apontam para a necessidade iminente de investir em aspectos estruturais dos serviços, de modo a potencializar a resolutividade da maioria dos problemas de saúde, seja por meio da garantia de condições mínimas para a execução de procedimentos corriqueiros, seja na qualificação profissional, para que estes sejam capazes de assegurar a identificação e a oferta de ações que atendam às mais diversificadas demandas de indivíduos e coletividades assistidas ${ }^{\mathbf{1 4}}$.

\section{Considerações finais}

A ESF configura-se como uma modalidade ambiciosa, inovadora e de incontestável relevância para o fortalecimento do SUS enquanto um sistema orientado à APS e comprometido com a qualidade e a equidade do acesso. $\mathrm{O}$ desenvolvimento de práticas avaliativas nesse contexto é fundamental, pois, à medida que possibilita o monitoramento e o aprimoramento dos serviços, favorece a implementação de uma APS com todas as potencialidades que esse modelo é capaz de proporcionar.

As disparidades observadas, referentes às percepções de profissionais e usuários, reforçam a importância de se incluírem no processo avaliativo opiniões dos diferentes interessados e/ou beneficiados do fenômeno avaliado, produzindo, assim, informações sob múltiplas perspectivas e mais condizentes com a realidade. Essas discrepâncias apontam, ainda, para a necessidade de se criarem nos serviços espaços mais dialógicos, que oportunizem a expressão dos anseios e opiniões, fortalecendo o controle social e a inclusão dos usuários no planejamento da assistência.

Os baixos valores verificados para a maioria dos atributos, sob a ótica de quem utiliza o serviço, evidenciam a dificuldade das ESF pesquisadas na efetivação dos atributos da APS e sinalizam a necessidade iminente de os serviços, continuamente, refletirem sobre suas práticas, a fim de identificarem as falhas e convergirem esforços para a reversão dos principais aspectos que repercutem negativamente no desempenho e na capacidade assistencial de suas equipes. Eles relevam, também, os desafios que municípios de médio porte enfrentam ainda hoje na consolidação e estruturação da ESF, tendo que se equilibrar, a 
partir de recursos cada vez mais escassos, entre o aumento progressivo dos gastos, gerados pela crescente demanda por atendimentos e procedimentos de média e alta complexidade, e a necessidade constante de investimentos para ampliação e qualificação da rede de APS em virtude da alta densidade populacional.

Os resultados apresentados nesta investigação inserem importantes implicações para os serviços avaliados acerca da sua congruência com relação aos atributos da APS. Todavia, menciona-se como limitação o fato de os resultados restringirem-se a um único município, o que, mesmo contribuindo para a caracterização do panorama nacional da qualidade das equipes de saúde da família, impossibilita sua generalização. Outra limitação refere-se ao instrumento adotado, que, embora constitua uma das ferramentas mais sensíveis e fidedignas para avaliação da qualidade de serviços de APS, não contempla a configuração multiprofissional das equipes de saúde nos moldes da ESF, abordando fundamentalmente aspectos relacionados à prática de profissionais médicos e enfermeiros.

\section{Colaboradores}

Ribeiro LA (0000-0003-0525-4758)* contribuiu para a concepção e planejamento; coleta e processamento dos dados; elaboração e revisão crítica do conteúdo; e para aprovação da versão final do manuscrito. Scatena JHG (0000-0002-7660-3479)* contribuiu para a concepção e planejamento; elaboração e revisão crítica do conteúdo; e para aprovação da versão final do manuscrito.

\section{Referências}

1. Giovanella L, Mendonça MHM. Atenção Primária à Saúde. In: Giovanella L, Escorel S, Lobato LVC, et al., organizadoras. Políticas e Sistema de Saúde no Brasil. Rio de Janeiro: Fiocruz; 2014. p. 493-546.

2. Silva JM, Caldeira AP. Modelo assistencial e indicadores de qualidade da assistência: percepção dos profissionais da atenção primária à saúde. Cad. Saúde Pública [internet]. 2010 [acesso em 2019 mar 15]; 26(6):1187-1193. Disponível em: http://www.scielo. br/pdf/csp/v26n6/12.pdf.

3. Andrade LOM, Bueno ICHC, Bezerra RC. Atenção Primária à Saúde e Estratégia Saúde da Família. In:
Campos GWS, Minayo MCS, Akerman M, et al., organizadores. Tratado de Saúde Coletiva. São Paulo: Hucitec; 2012. p. 783-836.

4. Brasil. Ministério da Saúde, Secretaria de Atenção à Saúde, Departamento de Atenção Básica. Política Nacional de Atenção Básica. Brasília, DF: MS, 2012.

5. Malta DC, Santos MAS, Stopa SR, et al. A Cobertura da Estratégia de Saúde da Família (ESF) no Brasil, segundo a Pesquisa Nacional de Saúde, 2013. Ciênc. Saúde Colet. [internet]. 2016 [acesso em 2019 jun 11]; 21(2):327-338. Disponível em: http://www.scielo.br/ pdf/csc/v21n2/1413-8123-csc-21-02-0327.pdf.
${ }^{*}$ Orcid (Open Researcher and Contributor ID). 
6. Viana ALd'A, Rocha JSY, Elias PE, et al. Modelos de atenção básica nos grandes municípios paulistas: efetividade, eficácia, sustentabilidade e governabilidade. Ciênc. Saúde Colet. [internet]. 2006 [acesso em 2019 jun 11]; 11(3):577-606. Disponível em: http:// www.scielo.br/pdf/csc/v1ln3/30976.pdf.

7. Escorel S, Giovanella L, Mendonça MHM, et al. O Programa de saúde da família e a construção de um novo modelo para a atenção básica no Brasil. Pan Am J Public Health [internet]. 2007 [acesso em $2019 \mathrm{fev}$ 12]; 21(2/3):164-176. Disponível em: https://scielosp. org/pdf/rpsp/2007.v2ln2-3/164-176/pt.

8. Costa NR. A Estratégia de saúde da família, a atenção primária e o desafio das metrópoles brasileiras. Ciênc. Saúde Colet. [internet]. 2016 [acesso em 2019 jun 11]; 21(5):1389-1398. Disponível em: http://www. scielo.br/pdf/csc/v21n5/1413-8123-csc-21-05-1389. pdf.

9. Brasil. Ministério da Saúde, Secretaria de Atenção à Saúde, Departamento de Atenção Básica. Manual Operacional da Segunda Etapa da Fase 2 do PROESF: Projeto de Expansão e Consolidação da Estratégia Saúde da Família. Brasília, DF: MS, 2012.

10. Portela GZ, Ribeiro JM. A sustentabilidade econômico-financeira da Estratégia Saúde da Família em municípios de grande porte. Ciênc. Saúde Colet. [internet]. 2011 [acesso em 2019 jun 11]; 16(3):1719-1732. Disponível em: http://www.scielo.br/pdf/csc/v16n3/09.pdf.

11. Neves RG, Flores TR, Duro SMS, et al. Tendência temporal da cobertura da Estratégia Saúde da Família no Brasil, regiões e Unidades da Federação, 2006-2016. Epidemiol. Serv. Saúde [internet]. 2018 [acesso em 2019 jun 3]; 27(3):e2017170. Disponível em: http:// www.scielo.br/pdf/ress/v27n3/2237-9622-ress-2703-e2017170.pdf.

12. Lavras C. Atenção primária à saúde e a organização de redes regionais de atenção à saúde no Brasil. Saúde soc. [internet]. 2011 [acesso em 2019 jul 15]; 20(4):867-874. Disponível em: http://www.scielo.br/ pdf/sausoc/v20n4/05.pdf.
13. Hauser L, Castro RCL, Vigo A, et al. Tradução, adaptação, validade e medidas de fidedignidade do Instrumento de Avaliação da Atenção Primária à Saúde (PCATool) no Brasil: versão profissionais de saúde. Rev. Bras. Med. Fam. Comunidade [internet]. 2013 [acesso em 2019 jun 29]; 8(29):244-55. Disponível em: https://rbmfc.org.br/rbmfc/article/view/821/584.

14. Starfield B. Atenção primária: equilíbrio entre necessidades de saúde, serviços e tecnologia. Brasília, DF: Unesco: Ministério da Saúde; 2002.

15. Brasil. Ministério da Saúde, Secretaria de Atenção à Saúde, Departamento de Atenção Básica. Manual do Instrumento de Avaliação da Atenção Primária à Saúde: Primary Care Assessment Tool - PCATool Brasil. Brasília, DF: MS, 2010.

16. Landsberg GAP, Souza Neto AI, Souza RA. Avaliando os atributos da atenção primária: uma revisão. Rev. Avaliação de Políticas Públicas [internet]. 2010 [acesso em 2018 dez 2]; 1(6):85-93. Disponível em: http:// www.avalrevista.ufc.br/index.php/revistaaval/article/view/70/92.

17. Fracolli LA, Gomes MFP, Nabão FRZ, et al. Instrumentos de avaliação da atenção primária à saúde: revisão de literatura e metassíntese. Ciênc. Saúde Colet. [internet]. 2014 [acesso em 2019 jul 15]; 19(12): 48514860. Disponível em: http://www.scielo.br/pdf/csc/ v19n12/pt_1413-8123-csc-19-12-04851.pdf.

18. D'Avila OP, Pinto LFS, Hauser L, et al. O uso do Primary Care Assessment Tool (PCAT): uma revisão integrativa e proposta de atualização. Ciênc. Saúde Colet. [internet]. 2017 [acesso em 2019 jul 15]; 22(3):855865. Disponível em: http://www.scielo.br/pdf/csc/ v22n3/1413-8123-csc-22-03-0855.pdf.

19. Kehrig RT, Martinelli NL, Spinelli MAS, et al. Antecedentes históricos da Regionalização da saúde em Mato Grosso. In: Scatena JHG, Kehrig RT, Spinelli MAS, organizadores. Regiões de saúde: diversidade e processo de regionalização em Mato Grosso. São Paulo: Hucitec; 2014. p. 111-133. 
20. Harzheim E, Pinto LF, Hauser L, et al. Avaliação dos usuários crianças e adultos quanto ao grau de orientação para atenção primária à saúde na cidade do Rio de Janeiro, Brasil. Ciênc. Saúde Colet. [internet]. 2016 [acesso em 2019 jul 15]; 21(5):1399-1408. Disponível em: http://www.scielo.br/pdf/csc/v2ln5/1413-8123csc-21-05-1399.pdf.

21. Carneiro MSM, Melo DMS, Gomes JM, et al. Avaliação do atributo coordenação da Atenção Primária à Saúde: aplicação do PCATool a profissionais e usuários. Saúde debate [internet]. 2014 [acesso em 2019 jul 15]; 38(esp):279-295. Disponível em: http:// www.scielo.br/pdf/sdeb/v38nspe/0103-1104-sdeb38-spe-0279.pdf.

22. Silva AS, Fracolli LA. Avaliação da Estratégia Saúde da Família: perspectiva dos usuários em Minas Gerais, Brasil. Saúde debate [internet]. 2014 [acesso em 2019 jul 15]; 38(103):692-705. Disponível em: http://www.scielo.br/pdf/sdeb/v38n103/0103-1104sdeb-38-103-0692.pdf.

23. Castro RCL, Knauth DR, Harzheim E, et al. Avaliação da qualidade da atenção primária pelos profissionais de saúde: comparação entre diferentes tipos de serviços. Cad. Saúde Pública [internet]. 2012 [acesso em 2019 jul 17]; 28(9):1772-1784. Disponível em: http://www.scielo.br/pdf/csp/v28n9/v28n9a15.pdf.

24. Silva AS. Avaliação dos atributos da atenção primária à saúde na Estratégia de Saúde da Família do Sul de Minas Gerais [tese]. São Paulo: Escola de Enfermagem. Universidade de São Paulo; 2014. 259 p.

25. Vitoria AM, Harzheim E, Takeda SP, et al. Avaliação dos atributos da atenção primária à saúde em Chapecó, Brasil. Rev. Bras. Med. Fam. Comunidade [internet]. 2013 [acesso em 2019 fev 16]; 8(29):285-93. Disponível em: https://rbmfc.org.br/rbmfc/article/ view/832/589.

26. Canesqui AM, Spinelli MAS. Saúde da família no estado de Mato Grosso, Brasil: perfis e julgamentos dos médicos e enfermeiros. Cad. Saúde Pública [internet]. 2006 [acesso em 2019 maio 22]; 22(9):1881-
1892. Disponível em: http://www.scielo.br/pdf/csp/ v22n9/12.pdf.

27. Medeiros CRG, Junqueira AGW, Schwingel G, et al. A rotatividade de enfermeiros e médicos: um impasse na implementação da Estratégia de Saúde da Família. Ciênc. Saúde Colet. [internet]. 2010 [acesso em 2019 jul 15]; 15(supl1):1521-1531. Disponível em: http://www.scielo.br/pdf/csc/v15sl/064.pdf.

28. Silva SA, Nogueira DA, Paraizo CMS, et al. Avaliação da Atenção Primária à Saúde: visão dos profissionais de saúde. Rev. esc. enferm. USP [internet]. 2014 [acesso em 2019 jun 26]; 48(esp):122-128. Disponível em: http://www.scielo.br/pdf/reeusp/v48nspe/pt_00806234-reeusp-48-esp-126.pdf.

29. Van Stralen CJ, Belisário SA, Van Stralen TBS, et al. Percepção dos usuários e profissionais de saúde sobre atenção básica: comparação entre unidades com e sem saúde da família na Região Centro-Oeste do Brasil. Cad. Saúde Pública [internet]. 2008 [acesso em 2019 abr 15]; 24(supl1):s148-s158. Disponível em: http://www.scielo.br/pdf/csp/v24sl/19.pdf.

30. Reis RS, Coimbra LC, Silva AAM, et al. Acesso e utilização dos serviços na Estratégia Saúde da Família na perspectiva dos gestores, profissionais e usuários. Ciênc. Saúde Colet. [internet]. 2013 [acesso em 2019 jul 15]; 18(11):3321-3331. Disponível em: http://www. scielo.br/pdf/csc/v18n11/22.pdf.

31. Silva AS, Baitelo TC, Fracolli LA. Avaliação da Atenção Primária à Saúde: a visão de usuários e profissionais sobre a Estratégia de Saúde da Família. Rev. Latino-Am. Enfermagem [internet]. 2015 [acesso em 2019 jun 26]; 23(5):979-87. Disponível em: http://www.scielo.br/pdf/ rlae/v23n5/pt_0104-1169-rlae-23-05-00979.pdf.

32. Oliveira MPR, Menezes IHCF, Sousa LM, et al. Formação e Qualificação de Profissionais de Saúde: Fatores Associados à Qualidade da Atenção Primária. Rev. Bras. Edu. Méd. [internet]. 2010 [acesso em 2019 maio 22]; 40(4):547-59. Disponível em: http://www.scielo. br/pdf/rbem/v40n4/1981-5271-rbem-40-4-0547.pdf. 
33. Harzheim E, Gonçalves MR, D’Avila OP, et al. Estudos de PCATool no Brasil. In: Mendonça MHM, Matta GC, Gondim R, et al. Atenção Primária à Saúde no Brasil: conceitos, práticas e pesquisa. Rio de Janeiro: Fiocruz; 2018. p. 493-524.

34. Chomatas E, Vigo A, Marty I, et al. Avaliação da presença e extensão dos atributos da atenção primária em Curitiba. Rev. Bras. Med. Fam. Comunidade [internet]. 2013 [acesso em 2013 dez 10]; 8(29):294-303. Disponível em: http://rbmfc.org.br/index.php/rbmfc/ article/view/828.

35. Ibañez N, Rocha JSY, Castro PC, et al. Avaliação do desempenho da atenção básica no estado de São Paulo. Ciênc. Saúde coletiva [internet]. 2006 [acesso em 2019 jul 15]; 11(3):683-703. Disponível em: http:// www.scielo.br/pdf/csc/v1ln3/30983.pdf.

36. Almeida MHM, Pacheco S, Krebs S, et al. Avaliação da atenção primária em saúde por usuários com e sem deficiência. CoDAS [internet]. 2017 [acesso em 2017 jan 23]; 29(5):e20160225. Disponível em: http:// www.scielo.br/pdf/codas/v29n5/2317-1782-codas29-5-e20160225.pdf.
37. Cunha EM, Giovanella L. Longitudinalidade/continuidade do cuidado: identificando dimensões e variáveis para a avaliação da Atenção Primária no contexto do sistema público de saúde brasileiro. Ciênc. Saúde Colet. [internet]. 2011 [acesso em 2019 maio 28]; 16(supl1):1029-1042. Disponível em: http://www. scielo.br/pdf/csc/v16sl/a36vl6sl.pdf.

38. Pereira MJB, Abrahão-Curvo P, Fortuna CM, et al Avaliação das características organizacionais e de desempenho de uma unidade de Atenção Básica à Saúde. Rev. Gaúcha Enferm. [internet]. 2011 [acesso em 2019 jul 15]; 32(1):48-55. Disponível em: http:// www.scielo.br/pdf/rgenf/v32nl/a06v32nl.pdf.

39. Silva CSO, Fonseca ADG, Souza LPS, et al. Integralidade e Atenção Primária à Saúde: avaliação sob a ótica dos usuários. Ciênc. Saúde Colet. [internet]. 2014 [acesso em 2019 jan 15]; 19(11):4407-4415. Disponível em: http://www.scielo.br/pdf/csc/v19n11/14138123-csc-19-11-4407.pdf.

Recebido em 18/07/2019

Aprovado em 22/11/2019

Conflito de interesses: inexistente

Suporte financeiro: não houve 